Conclusion There is evidence of change in practice already within the nursing staff approach to monitoring and managing confused patients. We plan to re-audit in October and introduce the same learning programme for all community staff especially Hospice at Home healthcare assistants to enhance detection of delirium within the home setting.

\section{P-130 IMPLANTABLE DEFIBRILLATOR DEACTIVATION: ATTITUDES AND KNOWLEDGE IN HEALTHCARE PROFESSIONALS}

1,2Lauren Brice, 'Susan Powell. 'University Hospitals Birmingham, Birmingham, UK; ${ }^{2}$ Marie Curie Hospice West Midlands, Solihull, UK

10.1136/bmispcare-2019-HUKNC.153

The prevalence of implantable defibrillator devices is increasing in the UK. These devices may prevent sudden cardiac death from arrhythmias, however, they do not alter disease trajectory in chronic conditions. Patients approaching end of life are predisposed to arrhythmias. This may lead to patients with implantable defibrillators receiving a shock in their final days of life which could be distressing and inappropriate when comfort is the priority. Despite this, elective deactivation of defibrillator devices is infrequently discussed and is often left until the last days of life.

An online survey of healthcare professionals was performed to assess attitudes toward device deactivation and knowledge of local procedures for deactivation. A total of 30 responses were collected. Responders were doctors and specialist nurses with variable clinical experience from a variety of specialties.

Only 50\% of individuals were able to correctly identify all implantable devices that contained a defibrillator component. Thirteen individuals felt they knew the protocol for arranging a planned deactivation of devices, however, only six individuals were able to correctly describe the process.

$77 \%$ of individuals felt that deactivation should be discussed as soon as possible in a patient identified as being in their last year of life and $73 \%$ felt that anyone involved in the patient's care should be able to have this discussion. In spite of this, when asked to rank their confidence having the conversation, the mean score was 54/100.

The most selected appropriate timescale between defibrillator deactivation and death from a chronic disease was months and $87 \%$ of individual felt that deactivation should occur in the outpatient setting or community rather than during an acute admission.

In summary, healthcare professionals felt that deactivation should be discussed and planned at the earliest opportunity, however, many did not feel confident having this discussion and were unable to identify all devices containing defibrillators or describe the local procedure for deactivation.

\section{P-131 INFORMATION WITH IMPACT: ANALYSIS OF FEEDBACK ABOUT WHAT TO EXPECT AT END OF LIFE ONLINE INFORMATION}

Amy Evans, Kim Bonnar, Morven Masterton. Marie Curie, London, UK
Background The Ambitions for Palliative and End of Life Care framework highlights the importance of information in supporting people affected by death and dying (National Palliative and End of Life Care Partnership, 2015). All information should meet high standards. Ensuring users can provide feedback is a core principle of the NHS Information Standard.

Aim A national charity sought feedback on its What to expect at end of life online information. The aim was to assess the usefulness of the information and to understand what worked, possible improvements, and any impact the information had on end of life experiences.

Method In March 2019 a survey was embedded on three web pages, which included two questions:

1. How useful did you find this page?

2. Please tell us why.

Results 106 responses were received. 88\% found the pages either Very useful (74\%) or Useful (14\%). Thematic analysis of the open text boxes identified these themes:

- Informative;

- Helped them prepare for future;

- Easy to understand;

- Sensitive/compassionate;

- Confidence/reassurance about actions.

The qualitative open text boxes provided examples of the impact the information can have, including:

'Having some idea of the indications of imminent death will help us to organise our time to ensure we're there when his time is approaching.'

'Although the situation is harrowing, I think part of the anxiety comes from fear of the unknown. This diminishes when we have the small comfort, of knowing what to expect.'

Six respondents felt there were gaps in the content, including how to deal with emotions.

Conclusion This research tells us that most respondents found the information useful, and that it has the potential to positively impact on people's end of life experiences.

\section{P-132 DRY MOUTH IN PALLIATIVE CARE, HOW ESSENTIAL OIL MOUTHWASH CAN HELP}

Alison Bailey. Weston Hospicecare, Weston Super Mare, UK

\subsection{6/bmjspcare-2019-HUKNC.155}

Background Dry mouth is extremely common in palliative care affecting 62\% of patients (Otukoya \& Doshi, 2018). To try and improve this for our patients a quality improvement programme (QIP) using essential oil mouthwash was agreed.

Aim To improve dry mouth for palliative patients using an evidence-based essential oil mouthwash, working in collaboration with medical and complementary therapy staff.

Method

- Precautions of using essential oils orally were identified;

- Patients were assessed for their suitability to use essential oils;

- All essential oils needed a safety data sheet and mouthwashes were clearly labelled;

- Observational study was undertaken 2018-2019;

- Staff trained to select sample group using validated assessment tool for inclusions (Health Education England: mouth care matters programme), exclusions: ulcers, thrush, patient unable to rinse, sensitive to oils, using oxygen; 
- Qualitative and quantitative data was analysed on day $1,4,8,12$;

- The amount and ingredients were standardised, and a doctor stated dosage and frequency on the patient drug chart;

- Dryness was scored from 1-10 (1 least -10 highest), and to state any comments.

Results Introducing the mouthwash has had a positive impact on patient care. 82 patients were referred across the services. Inpatients 49, Day hospice 9, Out patients 24 . $83 \%$ of the 82 participants continued stating positive comments compared to $17 \%$ who had to withdraw from the study. The average dryness score decreased from 7.4 start day 1 to 3.6 day 12 . Positive comments stated, 'Helps remove the metal taste after chemotherapy'; 'used at night don't have a dry mouth in morning'; 'helped after using inhaler'; 'refreshing better than others I have used' and 'if used before meals able to eat without feeling dry'.

Conclusion The results from patient feedback were qualitative and quantitative. Due to the volume of patients referred nurses were trained to prepare the mouthwash. Several hospices, care homes and the local hospital have shown an interest in this QIP due to the benefits patients expressed and want to use it for their patients. The positive impact for this change for our patients mean that we will continue to use the essential oil mouthwash.

\section{P-133 MOUTH CARE MATTERS: RAISING AWARENESS OF THE IMPACT OF DENTURE LOSS}

Connie Gillman, Victoria Cowell, Diana Lees. Mid Cheshire Hospitals, Crewe, UK

\subsection{6/bmispcare-2019-HUKNC.156}

Background Patients who rely on dentures do so not only for eating and communication but also as part of their identity and self-esteem. In 2017, denture losses in hospitals were estimated at 9,500 by the British Dental Association. This project was devised following the death of a patient who had lost her dentures during her final illness in hospital, which impacted upon her ability to eat and to communicate with her family and healthcare professionals.

Aims To assess the impact loss of dentures has on patients and to increase awareness amongst clinical staff regarding difficulties encountered by these patients.

Method A questionnaire was given to patients with dentures across two medical wards, inquiring:

- How loss of dentures would impact upon them?

- Had they been provided with a denture pot?

A 'Denture Reminder Form' was provided for attachment to the admission documentation with the aim of improving the use of denture pots.

Discussion

- $38 \%$ of patients with dentures were offered a denture pot, falling to $16 \%$ after implementation;

- $95 \%$ of patients felt it impacted their ability to communicate and $79 \%$ on their ability to eat;

- In 2018, 7 claims for replacement dentures were made costing $£ 969$.

Currently, the Trust has no standardised admission documentation making it difficult to ensure the form is regularly used, and there were instances of the form being discarded completely.
Healthcare professionals witness the impact on patients' quality of life, but have not recognised a simple intervention which can reduce denture losses together with the 'Mouth Care Matters' campaign (https://mouthcarematters.hee.nhs.uk/).

Conclusion We have 'one chance to get it right' (Department of Health, 2015) and for patients at the end of their lives, maintenance of their self-identity, self-esteem and ability to communicate and eat is as important as the medical care they receive. There are improvements that can and must be made to promote good oral care at the end of life.

\section{P-134 SUBCUTANEOUS LIDOCAINE INFUSION FOR COMPLEX PAIN IN HOSPICE INPATIENTS - A PRELIMINARY REPORT}

Jasmine Lee, Emma Hall, Rob George. St Christopher's Hospice, London, UK

\subsection{6/bmjspcare-2019-HUKNC.157}

Background Neuropathic pain is common and challenging to treat. Systemic lidocaine, a local anaesthetic, has established use in pain management (Eipe, Gupta \& Penning, 2016). Intermittent lidocaine infusions are safe, better than placebo and equally effective as other analgesics such as morphine (Challapalli, Tremont-Lukats, McNicol, Lau et al., 2005). Anecdotes of subcutaneous lidocaine infusions also report effectiveness in some cancer patients (Seah, Herschtal, Tran, Thakerar et al., 2017). To our knowledge use in palliative care patients has not been reported.

Aims

- To describe and evaluate subcutaneous lidocaine infusions in hospice inpatients;

- To assess from patients' and clinicians' observations if lidocaine is feasible, potentially effective, safe and tolerable.

Method Sequential retrospective review of hospice inpatients over seven months (November 2018 to May 2019) given subcutaneous lidocaine infusion for refractory pain. Patients were given a $2 \%$ lidocaine dose per 24 hours by CSCI. IPOS scores and documentary text were analysed at day 0,1 and 3 .

Results Twelve patients (eleven with malignancy) received lidocaine. All had complex pain, strong opioids and co-analgesia. Eight required anaesthetic intervention.

In seven pain scores improved. Drowsiness improved in seven. Two with worsening pain were close to death however, benefited in alertness. Overall two showed no improvement in either pain or drowsiness.

All tolerated lidocaine well for periods up to 122 days (median 7). One stopped prematurely due to incidental sepsis. One experienced opioid toxicity after dose increase which may be ascribed to lidocaine effects. One continued lidocaine at home and for $>4$ months.

Conclusions In this preliminary report, for patients with complex pain, refractory to standard multidrug regimes, lidocaine is a well-tolerated, safe, potential option for hospice inpatient use and supports the available anecdotal evidence. Observations suggest benefits in pain control and quality of life.

Lidocaine appears particularly valuable

- where patients are experiencing drowsiness from high frequency or high dose opioids;

- as an option pending anaesthetic intervention and;

- inpatients unable to swallow conventional neuropathics.

These findings justify a formal study which is under development. 